\title{
Preinoculation With the Probiotic Lactobacillus acidophilus Early in Life Effectively Inhibits Murine Citrobacter rodentium Colitis
}

\author{
CHIEN-CHANG CHEN, STEVE LOUIE, HAI NING SHI, AND W. ALLAN WALKER \\ Chang Gung Children's Hospital and Chang Gung University [C.-C.C.], Taoyuan, Taiwan; Mucosal \\ Immunology Laboratory [C.-C.C., S.L., H.N.S., W.A.W.], Massachusetts General Hospital and Harvard \\ Medical School, Charlestown, MA 02129
}

\begin{abstract}
ABSTRA
Enteropathogenic Escherichia coli (EPEC) is a common
pathogen in infantile diarrhea, causing a characteristic his-
topathologic attaching and effacing (A/E) lesion in the intestinal
mucosa. The mouse pathogen Citrobacter rodentium causes a
similar A/E lesion in the murine intestine. Like EPEC, $C$. roden-
tium infection results in colonic crypt hyperplasia, goblet cell
depletion, epithelial proliferation, and mucosal disruption. Using
this murine model, we tested the hypothesis that preinoculation
of murine gut with Lactobacillus acidophilus early in life can
enhance host defense against enteric bacterial infection and
attenuate bacteria-mediated colitis. Two-week old BALB/c mice
were inoculated with $L$. acidophilus twice per week for 4 weeks
before $C$. rodentium infection or concomitantly with the expo-
sure to $C$. rodentium at $6-8$ weeks of age. The probiotics were
administered twice weekly thereafter. We observed that $L$. aci-
dophilus inoculation in mice inhibits $C$. rodentium-induced co-
litis, which is associated with a decrease in $C$. rodentium colo-
nization and translocation, an increase in its clearance, and a
suppression of colonic myeloperoxidase (MPO) activity. Probi-
\end{abstract}
Infections caused by bacterial pathogens, such as enteropathogenic and enterohemorrhagic E. coli (EPEC and EHEC), represent a significant percentage of severe infantile diarrheas (1). EPEC and EHEC colonize the gastrointestinal mucosa by subverting intestinal epithelial cell function to produce a characteristic histopathological A/E lesion (2). The pathogenic features of EPEC or EHEC involve a disruption of the epithelial barrier and stimulation of a mucosal immune-mediated

Received January 14, 2005; accepted April 21, 2005.

Correspondence: W. Allan Walker, M.D., Mucosal Immunology Laboratory, Massachusetts General Hospital, 114 16th Street, 114-3503, Charlestown, MA 02129-4404: e-mail: wwalker@partners.org

Supported in part by a Hood Foundation Research Grant, a Career Development Award from the Crohn's and Colitis Foundation of America (to H.N.S.), by NIH grants RO1-DK070260, PO1-DK-33506 to Dr. Walker, and by the Clinical Nutrition Research Center at Harvard (P30-DK40561). Hai Ning Shi, B.V.M., Ph.D., is a recipient of an NIH KO1 Award (DK059996). Chien-Chang Chen, M.D., is sponsored by the Chang Gung University and Chang Gung Children's Hospital, Taoyuan, Taiwan.

DOI: 10.1203/01.pdr.0000183660.39116.83 otic treatment also stimulates regulatory cytokine expression in the colon [transforming growth factor $\beta$ (TGF- $\beta$ ), interleukin (IL)-10]. Preinoculation with L. acidophilus is more effective than concomitant use of probiotics in the induction of intestinal IgA secretion and in the downregulation of proinflammatory cytokine expression [tumor necrosis factor $\alpha$ (TNF- $\alpha$ ), IL-6, and IL-12]. These observations suggest that inoculation with probiotics can effectively prevent bacteria-induced colitis by limiting enteric bacteria infection and promoting mucosal protective regulatory immune responses. This study may have ramifications for prevention of infectious diarrhea in human infants and children, particularly in developing countries.(Pediatr Res 58: 1185-1191, 2005)

$\quad$ Abbreviations
MLN, mesenteric lymph node
MPO, myeloperoxidase
TGF- $\boldsymbol{\beta}$, transforming growth factor $\beta$
TNF- $\boldsymbol{\alpha}$, tumor necrosis factor $\alpha$

extensive inflammatory response. Similarly, the mouse enteric bacterial pathogen, $C$. rodentium, causes diarrhea, transmissible colonic hyperplasia, and colitis in mice as a consequence of its ability to colonize murine large intestinal enterocytes using the $\mathrm{A} / \mathrm{E}$ lesion formation (3-5). C. rodentium is the only known murine $\mathrm{A} / \mathrm{E}$ producing pathogen and therefore can serve as a small animal model for EPEC and EHEC infections.

Probiotics are live microorganisms that are ingested to promote beneficial effects on health by altering indigenous microflora. Probiotics have been used to prevent some intestinal pathogenic infections, such as Shigella, Salmonella, and enterohemorrhagic E. coli in murine models and enteropathogenic $E$. coli in a piglet model (6-9). It was also shown that probiotic treatment reduced $C$. rodentium-induced disease in adult mice (10). However, limited information is available regarding the effect of a probiotic inoculated during the preweaning stage on the subsequent host response to $C$. rodentium infection, which induces the intestinal pathologic alter- 
ations that are similar to those seen in many mouse models of colitis. Therefore, this model is an ideal model to study hostbacterial pathogen interactions in vivo (11) and can also be used to investigate the effect of probiotic inoculation on bacterial-induced intestinal diseases and on immune regulation of host responses to enteric infections.

The genus Lactobacillus, including at least 18 different species, has defined phenotypic and genotypic features in mice and humans (12-14). Probiotics have been considered a potentially important strategy to modulate inflammatory responses in the host gastrointestinal tract as they can enhance the host immune response and positively affect indigenous microflora in the host (15). L. acidophilus NCFM is a probiotic strain commercially available in the United States since the mid1970s and supplemented in some conventional foods such as milk, yogurt, and toddler formula. This strain survives gastrointestinal tract transit in both healthy and diseased populations and is associated with the reduction in severity of pediatric diarrhea (16). We hypothesized that 1) preinoculation with L. acidophilus early in life (before weaning) in mice will establish a better protective defense against $C$. rodentium infection than that of concurrent administration and 2) that inoculation with $L$. acidophilus would be beneficial in the intestinal microbial ecosystem and in the establishment of host intestinal epithelial and mucosal immune responses, contributing to an enhanced protection and an attenuation of Citrobacter-mediated intestinal injury.

\section{MATERIALS AND METHODS}

Mice and probiotic bacteria inoculation. Six- to 8-week-old female and male BALB/c ByJ mice were purchased from the Jackson Laboratory (Bar Harbor, ME) and bred in an animal facility at Massachusetts General Hospital. Neonatal mice were born to pregnant female Balb/c ByJ mice. Some mice were orally inoculated with L. acidophilus (Rhodia, Madison, WI) at 2 weeks of age for 4 weeks. All animal experiments were approved by the Institutional Animal Care and Use Committee. L. acidophilus were inoculated into deMan, Rogosa, and Sharpe broth (MRS; Difco, Detroit, MI) and grown at $37^{\circ} \mathrm{C}$ for 20 hours and then resuspended in PBS before being given orally to inoculate the mice (approximately $1 \times 10^{9} \mathrm{CFU}$ ).

C. rodentium infection and antigen preparation. Mice were orally inoculated with $C$. rodentium (strain DBS 100, from ATCC) at 6-8 weeks of age. Bacteria were grown overnight in Luria broth (LB) and resuspended in PBS before infecting the mice (approximately $5 \times 10^{8} \mathrm{CFU}$ ). C. rodentium antigen was prepared by collecting an overnight culture of $C$. rodentium in LB. The bacterial culture was washed in PBS and sonicated on ice. The homogenate was then centrifuged $(14,000 \mathrm{rpm})$ at $4^{\circ} \mathrm{C}$ for 30 minutes. Supernatants were collected and the protein concentration was determined.

Experimental design. Randomly selected neonatal mice born to pregnant female Balb/c ByJ mice were fed with probiotic bacteria beginning at 2 weeks of age. Group A was preinoculated with $L$. acidophilus from 2 weeks on. Group B was preinoculated with $L$. acidophilus from 2 weeks of age and infected with $C$. rodentium at 6-8 weeks. Group $C$ was concurrently inoculated with $L$. acidophilus and infected with $C$. rodentium on the same day at 6-8 weeks of age. Group D was infected with $C$. rodentium at 6-8 weeks of age without probiotic inoculation. After C. rodentium infection, L. acidophilus was administrated to groups $\mathrm{A}, \mathrm{B}$, and $\mathrm{C}$ twice per week throughout the experiment period. Group E consisted of normal controls. All mice were killed 7 or $14 \mathrm{~d}$ after $C$. rodentium infection. To assess the systemic effect of $C$. rodentium infection and probiotic inoculation, body weight and survival were measured throughout the experimental period.

Quantitation of C. rodentium and Lactobacillus acidophilus. To assess the clearance of $C$. rodentium, fecal pellets were collected from each mouse weekly. Fecal pellets were weighed, homogenized, serially diluted, and plated on selective MacConkey agar. To determine bacteria translocation, mice were killed at 7 and $14 \mathrm{~d}$ after $C$. rodentium infection. The MLN, spleen, and total segment of the colon were removed and homogenized in sterilized $1 \%$ Triton
100. An aliquot of the homogenate was cultured on MacConkey agar plates. Bacteria colonies were counted after overnight incubation. To assess the inoculating effect of the probiotic, fecal samples were also plated on Rogosa SL agar plates (Difco). After 72 hours of anaerobic incubation at $37^{\circ} \mathrm{C}$, bacteria colonies were identified and quantitated $(17,18)$.

Lymphocyte isolation. Mice were killed 2 weeks after $C$. rodentium infection. Lymphocyte suspensions were prepared from MLN and spleen as described previously (19). Cells $\left(5 \times 10^{6}\right.$ cells $\left./ \mathrm{mL}\right)$ were cultured on 24 -well plates in the presence or absence of $C$. rodentium antigen $(50 \mu \mathrm{g} / \mathrm{mL})$ or plate bound anti-CD3 MAb $(10 \mu \mathrm{g} / \mathrm{mL})$. Culture supernatants were collected 48 hours later and kept at $-20^{\circ} \mathrm{C}$ for future measurement of cytokine production.

Measurement of IFN- $\gamma$ and IL-10 production of MLN by ELISA. ELISA capture antibodies (R4-6A2, IFN- $\gamma$; JESS-2A5, IL-10) and biotinylated secondary antibodies (XMG1.2, IFN- $\gamma$; SXC-1, IL-10) were purchased from PharMingen (San Diego, CA). The biotinylated secondary antibodies were used as a second layer and reactions were visualized with $O$-phenylenediamine at $492 \mathrm{~nm}$ (OPD; Zymed Labs.). Standard curves were obtained using recombinant murine IFN- $\gamma$ (Genzyme) and IL-10 (R\&D Systems). OD values were converted to $\mathrm{pg} / \mathrm{mL}$ for each cytokine by linear regression with Delta Soft II (Biometallics, Princeton, NJ).

Fecal IgA antibody assays. Fecal pellets were collected into a protease inhibitor cocktail and were then weighed, homogenized, and incubated at $4^{\circ} \mathrm{C}$ for 1 hour. Supernatants were collected and stored at $-80^{\circ} \mathrm{C}$. Immuno II plates were coated with goat anti-mouse IgA $(2 \mu \mathrm{g} / \mathrm{mL}$; Southern Biotechnology Associates, Birmingham, AL) or C. rodentium antigen $(50 \mu \mathrm{g} / \mathrm{mL})$ and incubated overnight at $4^{\circ} \mathrm{C}$. Two rows in each plate used was coated with a goat anti-mouse IgA (Southern Biotechnology Associates) and used to generate a standard curve by using a standard mouse IgA (Southern Biotechnology Associates). Total IgA and antigen-specific IgA were detected using HRPconjugated anti-IgA as above. The reaction was developed with OPD (Zymed Labs) and read at $492 \mathrm{~nm}$.

Histopathological examinations. At necropsy, colonic tissues were isolated and small fragments were then frozen in tissue Tek OCT compound (Miles Inc. Elkhart, IN) and stored at $-80^{\circ} \mathrm{C}$. Some colonic fragments were snap frozen in liquid nitrogen and then stored at $-80^{\circ} \mathrm{C}$ for detection of cytokine expression and MPO activity. Five micrometer sections were cut on a 2800 Frigocut cryostat (Reichert-Jung, Germany) and stained with hematoxylin and eosin. Sections were analyzed without prior knowledge of treatment. Colonic pathology was scored according to a modified histologic scoring system previously published $(20,21)$. The scoring system consists of two parts: Part 1 was the determination of inflammatory cell infiltration in the colon, scored from 0 to 4 ( 0 : normal cells pattern; 1 : scattered inflammatory cells in the lamina propria; 2: increased numbers of inflammatory cells in the lamina propria; 3 : confluence of inflammatory cells extending into the submucosa; and 4: transmural extension of the infiltrative inflammatory cells). Part 2 was the evaluation of colon tissue damage which also ranged from 0 to 4 (0: normal tissue pattern; 1: discrete lymphoepithelial lesions; 2 : mild colonic crypt hyperplasia \pm focal invasion of epithelium; 3 : obvious colonic crypt hyperplasia, invasion of the epithelium and goblet cells depletion; 4: excessive mucosal damage extending through deeper structures of the bowel wall). The total colon pathology score was the summation of the inflammatory cell score and the tissue damage score.

Detection of colonic cytokine expression. Cytokine mRNA expression in colonic tissues was evaluated by real-time polymerase chain reaction (PCR). Total cellular RNA was isolated from frozen colonic tissue (distal part of the colon) using TRIzol (GIBCO Life Technologies) according to the manufacturer's instructions. cDNA was synthesized and subjected to real-time PCR. Mouse IL-6, TNF- $\alpha$, IL-12, IFN- $\gamma$, IL-10, TGF- $\beta$, and GAPDH real-time PCR probes, and primer pairs were purchased from Biosource International, Inc. (Camarillo, CA). Amplification of GAPDH was included for each experimental sample as an endogenous control. All experimental samples were amplified in duplicate.

MPO assay. MPO level was measured using a modified kinetic assay as described (22). Colonic segments were homogenized in $1 \%$ hexadecyl trimethylammonium in phosphate buffer to negate pseudoperoxidase activity. MPO activity was measured in supernatants following three cycles of sonication, freezing, thawing, and centrifuging at $13200 \mathrm{rpm}$ at $4^{\circ} \mathrm{C}$ for 15 minutes. The supernatant was mixed with potassium phosphate buffer containing 0.167 $\mathrm{mg} / \mathrm{mL} O$-dianiside dihydrochloride (Sigma Chemical Co.) and $0.0005 \%$ hydrogen peroxide. Activity was measured at $450 \mathrm{~nm}$.

Statistic analysis. All the results are expressed as the mean \pm SEM. N refers to the number of mice used. Statistical differences were determined using the two-tailed $t$ test or one-way analysis of variance test with SPSS software (SigmaStat; SPSS, Chicago, IL); $p<0.05$ was considered significant. 


\section{RESULTS}

C. rodentium-induced colonic pathology in mice with and without probiotic inoculation. As expected, mice exposed to $C$. rodentium alone showed signs of disease early in the infection such as soft stools, a hunched posture, disturbed body hair, and body weight loss. In contrast, mice pretreated with L. acidophilus started at 2 weeks of age or given concurrently with $C$. rodentium developed less severe disease resulting in decreased body weight loss during the course of the experiment (data not shown) compared with mice infected with $C$. rodentium. Histologic examination of the distal part of the colon in mice infected with $C$. rodentium alone showed the typical pathologic changes associated with this bacterial infection including thickening of the wall of the colon, colonic crypt hyperplasia, an extensive inflammatory cellular infiltration and disruption of the epithelial surface (Fig. 1D). Histologic analysis of colonic tissue from mice treated concurrently (Fig. 1C) or preinoculated (Fig. 1B) showed a less severe pathology compared with $C$. rodentium-infected mice alone (Fig. $1 D$ ), including a mild colonic crypt elongation, less cellular infiltration of the colonic lamina propria, a mild uneven epithelial

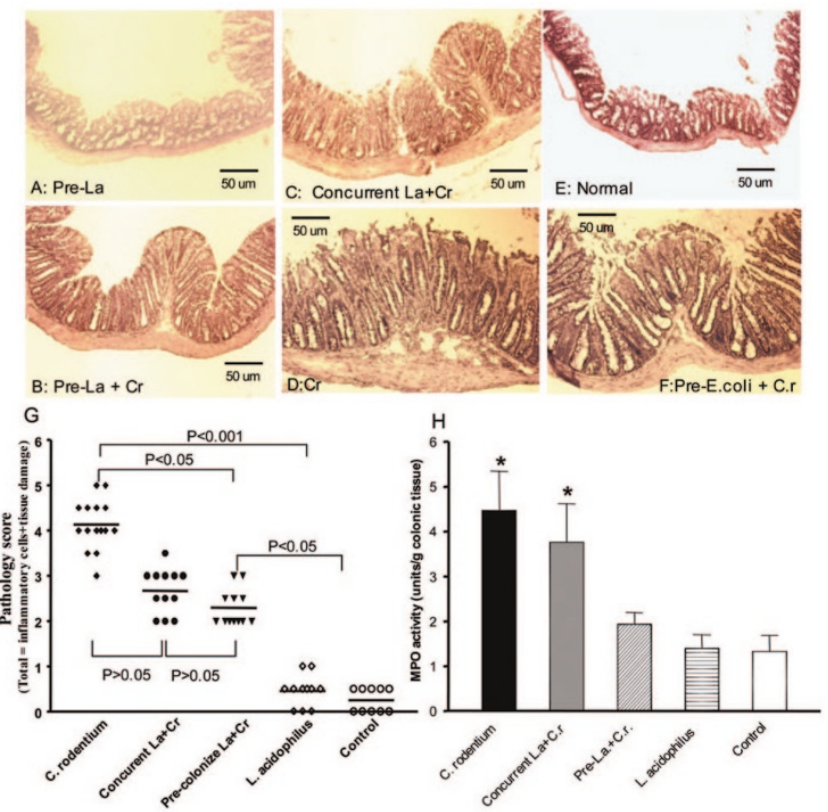

Figure 1. Representative histology of the distal colon 2 weeks after a $C$. rodentium infection. Colon tissue was prepared from the mice inoculated with L. acidophilus NCFM only (A) or preinoculated with $L$. acidophilus NCFM and then infected with $C$. rodentium $(B, \mathrm{PreLa}+\mathrm{Cr}$ ), concurrently administered with $L$. acidophilus NCFM and $C$. rodentium $(C$, concurrent $\mathrm{La}+\mathrm{Cr}), C$. rodentium-infected $(D)$, untreated control $(E)$, and $E$. coli-preinoculated and $C$. rodentium-infected $(E)$ and stained with hematoxylin and eosin (magnification $\times 100)$. $(G)$ The colonic pathology score of different groups of mice at 2 weeks after $C$. rodentium infection. The scores were assessed by determination of inflammation and tissue damage. The figures shown are measurements of individual mice pooled from three independent experiments. The horizontal line represents the mean score of different groups. Data from the colonic pathology scores was analyzed using one-way analysis of variance (nonparametric). (H) MPO activity was measured in the supernatants of homogenated colonic tissue. The data shown are the level of MPO activity per gram of colonic tissue at 2 weeks after $C$. rodentium infection. The data are represented of the mean \pm SEM and statistical significant differences compared with healthy control group: $* p<0.05(n=10-15)$. surface versus the control (Fig. 1E). Furthermore, the inflammation and intestinal damage scores $(20,21)$ were significantly lower in preinoculated mice than those with $C$. rodentium infection alone (Fig. $1 G$ ) versus controls. In addition, a comparable colonic inflammation was also observed in the BLAB/c mice when preinoculated with a commensal $E$. coli twice weekly and then infected with $C$. rodentium compared with the mice infected with $C$. rodentium alone (Fig. $1 F$ ).

MPO activity in the intestine can be used to quantitate inflammation $(22,23)$. In this study, the impact of pre- and concurrent inoculation with probiotics on colonic inflammation was examined by measuring MPO activity of colonic tissues. Our results showed that mice infected with $C$. rodentium (pooled $n=15$ ) and mice concurrently inoculated with probiotic $(n=18)$ had significantly higher MPO levels compared with healthy control mice $(n=10)$, suggesting that $C$. rodentium infection induces the infiltration of inflammatory cells into the colon and that concurrent inoculation with the probiotics at the adult stage has little or no effect on colonic MPO activity. In contrast, mice that were preinoculated with $L$. acidophilus early in life showed significantly decreased levels of MPO in the colon, suggesting an effect of probiotic in attenuating the bacteria-mediated inflammatory response in the colon. (Fig. $1 H$ ). Taken together, our results indicated that at an adult stage, a concurrent inoculation of probiotics and $C$. rodentium was unable to reduce MPO activity in the colon, whereas probiotic inoculation at an early age resulted in a reduced MPO activity in the intestine, contributing to the attenuation of the bacteriainduced intestinal inflammation.

Probiotics inhibit colonization, proliferation, and translocation of $C$. rodentium. We then determined whether $L$. acidophilus pre- and concurrent inoculation affected colonization, proliferation, and clearance of $C$. rodentium. At 1 and 2 weeks after $C$. rodentium infection, fecal pellets were collected, homogenized, and plated onto selective MacConkey agar plates. Our results showed that bacterial counts were lower in L. acidophilus-preinoculated mice (Fig. $2 A$ ) at an early stage of infection (1 week post-infection). A significantly lower level of bacteria shed in feces was also detected in L. acidophiluspreinoculated mice by the second week after infection (Fig. $2 B$ ), suggesting that the probiotic treatment reduced proliferation of $C$. rodentium. The intermediate levels of bacteria recovery from mice that were concurrently inoculated with L. acidophilus and C. rodentium at both time points (Fig. 2A, $B$ ) indicated that concurrent inoculation with the probiotic was less effective in altering establishment and persistence of $C$. rodentium infection in mice than preinoculation of $L$. acidophilus. An effective colonization by L. acidophilus was maintained by twice- weekly inoculation and confirmed by a series of stable bacterial counts on Rogosa SL agar plates (data not shown).

We next determined whether probiotic pre- and concurrent inoculation would have a beneficial effect on epithelial barrier function by examining $C$. rodentium mucosal transmigration in probiotic-treated infected and control mice. One and 2 weeks following $C$. rodentium infection, at the peak of inflammation, we killed mice and collected MLN, spleen, and total colonic segments from each mouse to assess bacterial load. We found 

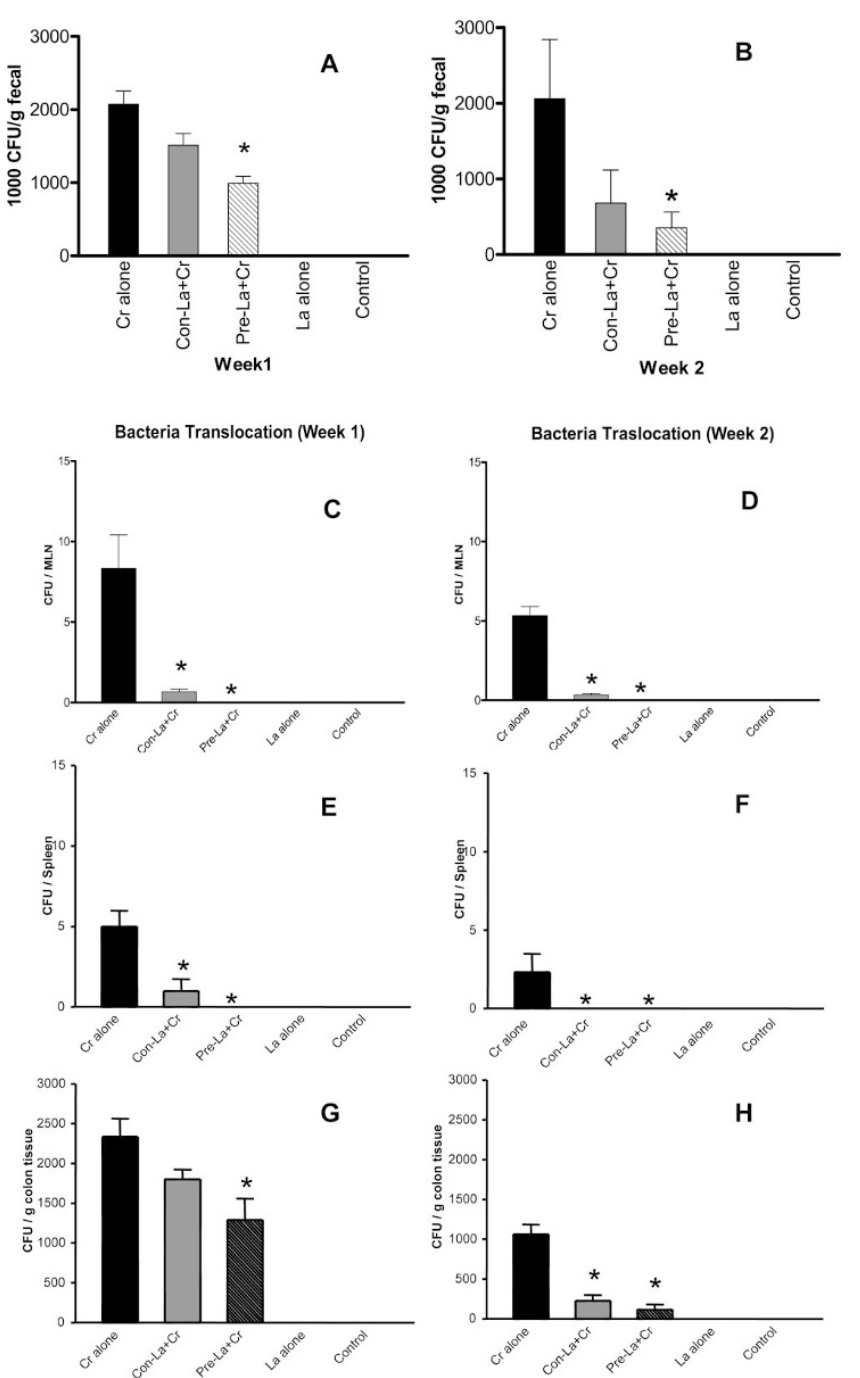

Figure 2. Analysis of fecal C. rodentium counts as determined by a selective MacConkey agar plate. The data shown are the number of $C$. rodentium recovered per gram of fecal samples from mice of different groups at 1 week $(A)$ and 2 weeks $(B)$ after $C$. rodentium infection. The data are represented as the mean $\pm \operatorname{SEM}(n=10-16$ at each time point). Viable $C$. rodentium recovered in the MLN $(C, D)$ spleen $(E, F)$ and colonic tissue $(G, H)$ at 1 and 2 weeks after $C$. rodentium infection. At necropsy, the MLN, spleen, and colonic tissue were removed and homogenized. An aliquot of diluted homogenized tissue was plated on selective MacConkey agar plates and incubated overnight. Viable bacteria were counted. The data are represented as the mean \pm SEM ( $n=5-10$ at each time point $)$ and statistical significant differences compared with the $C$. rodentium-alone group: ${ }^{*} p<0.05$.

that there were significantly fewer viable $C$. rodentium bacteria recovered from the MLN (Fig. 2C) and spleen (Fig. 2E) of both L. acidophilus pre- and concurrently inoculated mice 1 week after infection compared with mice infected with $C$. rodentium alone. At 2 weeks after $C$. rodentium infection, the number of $C$. rodentium bacteria recovered from MLN (Fig. 2D) and spleen (Fig. $2 F$ ) of mice infected with $C$. rodentium alone was similar to that detected the first week, whereas $L$. acidophilus inoculated mice (both pre- and concurrently) had significantly lower (or no) counts. These results suggest that pre- and concomitant treatment with probiotics may enhance colonic epithelial barrier function, resulting in a decrease in the entry of luminal bacteria across the colonic mucosa. There were also fewer viable $C$. rodentium bacteria recovered from the total colonic segments of mice both pre- and concomitantly treated with Lactobacillus 1 week (Fig. 2G) and 2 weeks (Fig. 2H) after infection compared with those of mice infected with $C$. rodentium alone. This suggests that probiotic treatment in mice inhibits $C$. rodentium colonization and promotes the clearance of $C$. rodentium from mouse intestine.

Probiotic pre- but not concurrent inoculation enhances IgA secretion in the intestinal lumen Our examination of the impact of probiotics on the host's intestinal $\operatorname{IgA}$ response showed that mice with $L$. acidophilus preinoculation alone $(60 \pm 2.0$ $\mu \mathrm{g} / \mathrm{mL}$ ) and $L$. acidophilus preinoculation plus C. rodentium infection $(63 \pm 3.5 \mu \mathrm{g} / \mathrm{mL})$ had higher total IgA levels compared with the other three groups (range, $20-41 \mu \mathrm{g} / \mathrm{mL}$ ). Mice preinoculated with $L$. acidophilus early in life had significantly higher levels $(8.2 \pm 0.2 \mu \mathrm{g} / \mathrm{mL}, p<0.05)$ of anti-C. rodentium specific IgA than mice with $C$. rodentium infection alone (4.1 $\pm 0.1 \mu \mathrm{g} / \mathrm{mL}$ ) or $C$. rodentium plus concomitant probiotic administration $(4.7 \pm 0.2 \mu \mathrm{g} / \mathrm{mL})$.

Probiotic pre- and concurrent inoculation alters cytokine responses in the intestinal mucosa. To examine the influence of $L$. acidophilus treatment on the pathogen-induced cytokine response in intestinal mucosa, we examined antigen-specific cytokine production in MLN. We found that both pre- and concomitant inoculation with Lactobacillus enhanced the C. rodentium antigen-specific IFN- $\gamma$ (Fig. $3 A$ ) and IL-10 (Fig. $3 B$ ) responses and that preinoculation showed a more significant impact than concurrent treatment on the response of these cytokines.

To further examine the contribution of probiotic inoculation on the modulation of the local cytokine response in the intestine, we examined the expression of both proinflammatory and immune regulatory cytokines in the colon by real-time PCR. Our results show that $C$. rodentium infection induces proinflammatory cytokine (TNF- $\alpha$ and IL-6) expression (Fig. 4A,B), and that administration of $L$. acidophilus either alone or concurrently with $C$. rodentium results in a downregulation of the colonic TNF- $\alpha$ and IL-6 response (Fig. 4A,B). We have observed that preinoculation with $L$. acidophilus results in an enhanced colonic IFN- $\gamma$ expression (Fig. $4 C$ ) and that concurrent inoculation with $L$. acidophilus reduces IFN- $\gamma$ expression in colonic tissue. Our results also showed a significant increase in colonic IL-12 expression in C. rodentium-infected mice and
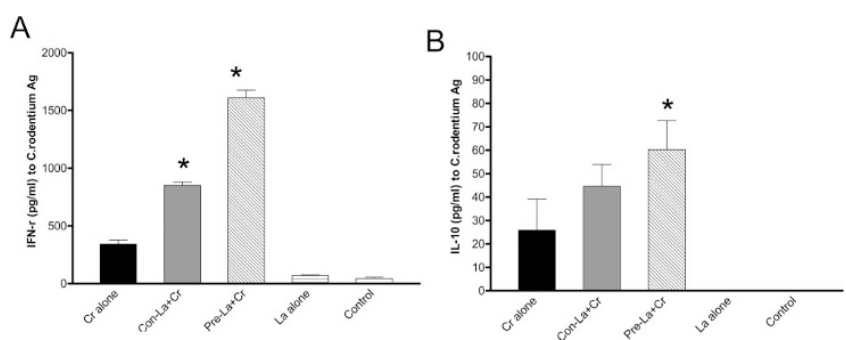

Figure 3. IFN- $\gamma(A)$ and IL-10 $(B)$ production by MLN cells from $C$. rodentium-infected and probiotic treated groups at 2 weeks after $C$. rodentium infection. MLN cells were collected and restimulated with $C$. rodentium antigen $(50 \mu \mathrm{g} / \mathrm{mL})$ for 48 hours. Cytokine secreted into culture supernatants was measured by ELISA. The results are expressed as the mean \pm SEM and are representative of three independent experiments. ${ }^{*} p<0.05, n=5$ mice. 

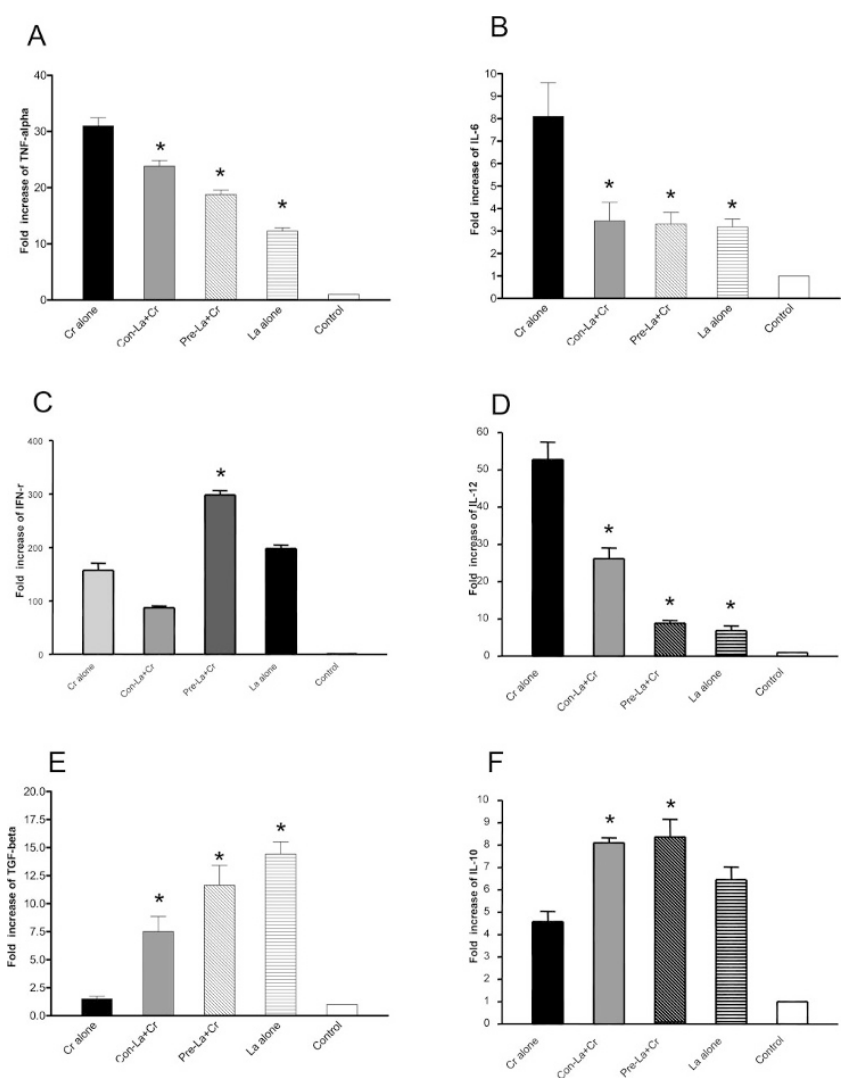

Figure 4. Cytokine mRNA expression in colonic tissue was measured by real-time PCR at 2 weeks after $C$. rodentium infection. Values are the mean fold increase compared with baseline obtained from control animals. (A) TNF- $\alpha,(B)$ IL-6, $(C)$ IFN- $\gamma,(D)$. IL-12, $(E)$ TGF- $\beta$, and $(F)$ IL-10. The data shown are from one of two experiments performed, showing similar results. Statistical significant differences compared with the $C$. rodentium-alone group: ${ }^{*} p<0.05, n=5$ mice per group.

that probiotic pre- and concomitant inoculation significantly reduced the $C$. rodentium-associated colonic IL-12 response (Fig. 4D).

Immune regulatory cytokines, such as IL-10 and TGF- $\beta$, are known to play a role in suppression of intestinal inflammation. To determine whether pre- and concurrent inoculation with probiotics could alter regulatory immune responses in the intestinal mucosa, we compared mRNA expression levels of TGF- $\beta 1$ and IL-10 among different groups. The results presented in Fig. $4 E$ demonstrate that probiotic treatment (both pre- and concurrent) significantly upregulates colonic TGF- $\beta 1$ expression, which is absent in the colon of the mice infected with $C$. rodentium alone. Both probiotic pre- and concomitant inoculated mice showed an enhanced colonic IL-10 expression (Fig. $4 F$ ) compared with mice infected with $C$. rodentium alone. Taken together, our results suggest a role for probiotics in immune regulation during enteric bacterial infection.

\section{DISCUSSION}

Previous studies have demonstrated inhibitory effects of probiotic use on pathogenic bacteria in the intestinal tract. Most of these studies have shown that short-term probiotic administration at the time of infection can shorten and/or reduce the severity of the resultant disease course. Although it was also reported previously that administration of L. acidophilus attenuated $C$. rodentium-induced disease in adult mice (10), few studies have addressed the potential preventive effect of probiotics used during the preweaning stage on subsequent exposure to intestinal pathogens. In this study, we tested the hypothesis that preinoculation of murine gut with $L$. acidophilus early in life (i.e. during the time of immaturity of the intestinal immune system) will have profound consequences in host defense against subsequent enteric bacterial infection later in life.

Probiotics have been shown to be effective in inhibiting intestinal bacterial infection (6-9) and intestinal inflammation, such as dextran sulfate sodium- and trinitrobenzene sulfonic acid-induced colitis (24-27) in animal models. Multiple mechanisms by which probiotics enhance protection against pathogens have been suggested including stimulation of pathogenspecific immunity, competition for limited nutrients, inhibition of pathogen mucosal adherence, and invasion and production of antimicrobial substances (28). Our data suggested that inoculation with L. acidophilus NCFM could effectively inhibit C. rodentium-associated colitis. The protection activated by probiotic use in $C$. rodentium-infected mice correlates with reduced bacterial loads in the gut, enhanced mucosal immune responses evidenced by an increase in mucosal IFN- $\gamma$ production and IgA secretion, and a downregulation of the proinflammatory cytokine response. Moreover, our results provide evidence suggesting that probiotic colonization induces an immune regulatory response, which may also be involved in suppression of bacteria-induced tissue injury. In an animal study in which a fermented mixture containing L. acidophilus $\left(10^{5}\right.$ cells $\left./ \mathrm{mL}\right)$ was fed to mice with $E$. coli-induced diarrhea, this measure helped to decrease the diarrhea (29). In a bovine model in which probiotic-treated calves were either given $E$. coli $\mathrm{O} 157: \mathrm{H7}$ or E. coli O111: NM, fecal shedding was reduced compared with that for untreated infected calves (30). Shu et al. (31) also found that in a piglet model dietary treatment using probiotics could reduce the severity of weaning diarrhea associated with rotavirus and $E$. coli and was thought to be due to an enhanced immune-mediated protection.

The major finding of our study was that preinoculation with L. acidophilus NCFM significantly altered the dynamics of $C$. rodentium infection in the colon. In general, $C$. rodentium resides primarily in an extracellular location on the epithelial surface (32) and in the lamina propria or edematous submucosa (33). Our results show that inoculation with L. acidophilus, particularly preinoculation, in mice reduced $C$. rodentium infection, inhibited its proliferation, and facilitated its clearance. Such an impact of probiotics on $C$. rodentium infection is found to be more pronounced in mice with preinoculation of $L$. acidophilus, indicating a better protection than concurrent administration of the probiotic. Moreover, these preinoculated mice also prevented local or systemic spread of infection as evidenced by a decrease in bacterial translocation (e.g. no bacteria found in the MLN and spleen). This agrees with a previous study showing that feeding live Lactobacillus plantarum or Lactococcus lactis inhibited translocation of an administered bacteria in healthy as well as in TNBS-treated mice (26). Thus, our study and those discussed above demonstrate 
that probiotics can enhance host defense by decreasing pathogenic bacterial loads, which acts to further protect the mucosal from invasion.

The inoculation with probiotics has been shown to increase the antibody response to gut pathogens $(34,35)$. Our data show that mice inoculated with $L$. acidophilus produced significantly more total and antigen-specific IgA, which was accompanied by a less severe $C$. rodentium-induced colitis. This observation is in line with previous studies showing a significantly higher level of intestinal antibacterial $\operatorname{IgA}$ responses (36) among probiotic-fed mice and enhanced specific IgA to bacterial toxin in mice fed with yogurt-containing probiotics compared with controls (37). In an animal study, mice fed bifidobacteria for $12 \mathrm{~d}$ showed significantly higher levels of fecal total $\operatorname{IgA}$ compared with that of the control group (38). The above results suggest that probiotics can enhance local production of IgA in the intestine. However, the role of antibody production in host defense against $C$. rodentium infection is still inconclusive. Using various types of knockout mice, a recent study evaluated the importance of secreted antibodies ( $\operatorname{IgA}, \operatorname{IgM}$, and $\operatorname{IgG})$ and B cells in host defense against $C$. rodentium infection and found that $\operatorname{IgG}$, but not $\operatorname{IgA}$ or $\operatorname{IgM}$, antibodies are important in host protection against $C$. rodentium infection (39).

In this study we observed that $L$. acidophilus inoculation can activate colonic regulatory cytokine responses. The capacity of L. acidophilus to regulate intestinal cytokine responses is demonstrated by the results from the real-time PCR experiments showing that probiotic inoculation induces an upregulation of regulatory/anti-inflammatory cytokines and a downregulation of proinflammatory cytokine expression in the colon. These results may suggest the contribution of probioticinduced immune regulatory responses in the reduction of $C$. rodentium-mediated colitis. IL-10 and TGF- $\beta$ are regulatory cytokines produced by specialized subsets of $\mathrm{T}$ helper cells, which have anti-inflammatory activities. IL-10 might also have an indirect anti-inflammatory effect because it also stimulates together with TGF- $\beta$ IgA synthesis (40). The probiotic Lactobacillus paracasei has been shown to maintain IL-10 and induce TGF- $\beta$ secretion by CD4+ T cells (41). An in vitro study has demonstrated that the incubation of the probiotic $L$. plantarum with mononuclear cells isolated from actively inflamed colon results in a significant increase of IL-10 (42). Our results were further supported by a recent study that showed that probiotic treatment was able to ameliorate the severity of TNBS-induced colitis by inducing an immune-regulatory response involving TGF- $\beta$ regulatory cells (43). In this study we also observed that infection with $C$. rodentium resulted in a reduction in a $L$. acidophilus-induced TGF- $\beta$ response. Although the mechanism by which $C$. rodentium modifies host cytokine responses is still unknown, it may be possible that $C$. rodentium or its products have the capacity to inhibit certain host cytokine responses as suggested by a previous study (43).

Our results also show that $L$. acidophilus inoculation leads to a downregulation of the proinflammatory cytokine TNF- $\alpha$ and IL-6 mRNA expression as well as IL-12 mRNA levels in the colon. In a previous study, TNF- $\alpha$ levels and expression of their mRNA were decreased in mice treated with a Bifidobacterium longum HY8001 culture supernatant (44). It has been reported that Lactobacillus species can generate soluble molecules that inhibit TNF- $\alpha$ production in activated macrophages (45). Dendritic cells were unresponsive to $L$. plantarum and Bifidobacterium adolescentis but produced large amounts of IL-12p70, TNF- $\alpha$, and IL-6 in response to E. coli (46). Our results also show the complexity of intestinal cytokine regulation by probiotics as evident by the opposite trend in the colonic IFN- $\gamma$ response with an upregulation in pretreated and downregulation in concurrently inoculated mice. Although the Th1 response has been suggested to play a protective role in C. rodentium infection (47), the implications and mechanisms for the differentially altered colonic IFN- $\gamma$ response observed in a current study are unclear and need to be further studied. Taken together, our results suggest that the probiotic L. acidophilus may have a role in controlling local inflammation by modulating the cytokine environment in the infected intestine.

In summary, our investigation of a prophylactic approach for bacteria-induced colitis in mice involving inoculation with L. acidophilus has shown that probiotic inoculation results in an attenuation of $C$. rodentium-mediated colonic pathology. Our results also showed that long-term preinoculation early in life had a better protective effect than concurrent administration of probiotics as evidenced by a more pronounced inhibitory effect on pathogenic bacterial proliferation and proinflammatory cytokine expression and by a more effective stimulatory effect on protective and regulatory immune responses to the enteric bacterial pathogen $C$. rodentium. These results may have medical implications in the prophylaxis and management of bacteria-induced diarrhea and intestinal inflammation in children and even adults at risk.

Acknowledgment. The authors are grateful to Dr. Beth McCormick for critical review of the manuscript.

\section{REFERENCES}

1. Vallance BA, Finlay BB 2000 Exploitation of host cells by enteropathogenic Escherichia coli. Proc Natl Acad Sci U S A 97:8799-8806

2. Moon HW, Whipp SC, Argenzio RA, Levine MM, Giannella RA 1983 Attaching and effacing activities of rabbit and human enteropathogenic Escherichia coli in pig and rabbit intestines. Infect Immun 41:1340-1351

3. Schauer DB, Falkow S 1993 The eae gene of Citrobacter freundii biotype 4280 is necessary for colonization in transmissible murine colonic hyperplasia. Infect Immun 61:4654-4661

4. Ghaem-Maghami M, Simmons CP, Daniell S, Pizza M, Lewis D, Frankel G, Dougan G 2001 Intimin-specific immune responses prevent bacterial colonization by the attaching-effacing pathogen Citrobacter rodentium. Infect Immun 69:5597-5605

5. Frankel G, Phillips AD, Novakova M, Field H, Candy DC, Schauer DB, Douce G, Dougan G 1996 Intimin from enteropathogenic Escherichia coli restores murine virulence to a Citrobacter rodentium eaeA mutant: induction of an immunoglobulin A response to intimin and EspB. Infect Immun 64:5315-5325

6. Nader de Macias ME, Apella MC, Romero NC, Gonzalez SN, Oliver G 1992 Inhibition of Shigella sonnei by Lactobacillus casei and Lact. Acidophilus. J Appl Bacteriol 73:407-411

7. Filho-Lima JV, Vieira EC, Nicoli JR 2000 Antagonistic effect of Lactobacillus acidophilus, Saccharomyces boulardii and Escherichia coli combinations against experimental infections with Shigella flexneri and Salmonella enteritidis subsp. Typhimurium in gnotobiotic mice. J Appl Microbiol 88:365-370

8. Shu Q, Gill HS 2002 Immune protection mediated by the probiotic Lactobacillus rhamnosus HN001 (DR20) against Escherichia coli O157:H7 infection in mice. FEMS Immunol Med Microbiol 34:59-64

9. Asahara T, Shimizu K, Nomoto K, Hamabata T, Ozawa A, Takeda Y 2004 Probiotic bifidobacteria protect mice from lethal infection with Shiga toxin-producing Escherichia coli $\mathrm{O} 157: \mathrm{H} 7$. Infect Immun 72:2240-2247

10. Varcoe JJ, Krejcarek G, Busta F, Brady L 2003 Prophylactic feeding of Lactobacillus acidophilus NCFM to mice attenuates overt colonic hyperplasia. J Food Prot 66:457-465

11. MacDonald TT, Frankel G, Dougan G, Goncalves NS, Simmons C Host defences to Citrobacter rodentium. Int J Med Microbiol 2003;293:87-93 
12. Kimura K, McCartney AL, McConnell MA, Tannock GW 1997 Analysis of fecal populations of bifidobacteria and lactobacilli and investigation of the immunological responses of their human hosts to the predominant strains. Appl Environ Microbiol 63:3394-3398

13. Ahrne S, Nobaek S, Jeppsson B, Adlerberth I, Wold AE, Molin G 1998 The norma Lactobacillus flora of healthy human rectal and oral mucosa. J Appl Microbiol 85:88-94

14. Walter J, Hertel C, Tannock GW, Lis CM, Munro K, Hammes WP 2001 Detection of Lactobacillus, Pediococcus, Leuconostoc, and Weissella species in human feces by using group-specific PCR primers and denaturing gradient gel electrophoresis. Appl Environ Microbiol 67:2578-2585

15. Cong Y, Konrad A, Iqbal N, Elson CO 2003 Probiotics and immune regulation of inflammatory bowel diseases. Curr Drug Targets Inflamm Allergy 2:145-154

16. Sanders ME, Klaenhammer TR 2001 Invited review: the scientific basis of Lactobacillus acidophilus NCFM functionally as a probiotic. J Dairy Sci 84:319-331

17. Varcoe J, Zook C, Sui J, Leighton S, Busta F, Brady L 2002 Variable response to exogenous Lactobacillus acidophilus NCFM consumed in different delivery vehicles. J Appl Microbiol 93:900-906

18. Sui J, Leighton S, Busta F, Brady L 2002 16S ribosomal DNA analysis of the faeca lactobacilli composition of human subjects consuming a probiotic strain Lactobacillus acidophilus NCFM. J Appl Microbiol 93:907-912

19. Shi HN, Liu HY, Nagler-Anderson C 2000 Enteric infection acts as an adjuvant for the response to a model food antigen. J Immunol 165:6174-6182

20. Burns RC, Rivera-Nieves J, Moskaluk CA, Matsumoto S, Cominelli F, Ley K 2001 Antibody blockade of ICAM-1 and VCAM-1 ameliorates inflammation in the SAMP $1 /$ Yit adoptive transfer model of Crohn's disease in mice. Gastroenterology 121:1428-1436

21. Loher F, Schmall K, Freytag P, Landauer N, Hallwachs R, Bauer C, Siegmund B, Rieder F, Lehr HA, Dauer M, Kapp JF, Endres S, Eigler A 2003 The specific type-4 phosphodiesterase inhibitor mesopram alleviates experimental colitis in mice. J Pharmacol Exp Ther 305:549-556

22. Miller MJ, Sadowska-Krowicka H, Chotinaruemol S, Kakkis JL, Clark DA 1993 Amelioration of chronic ileitis by nitric oxide synthase inhibition. J Pharmacol Exp Ther 264:11-16

23. Wallace JL, MacNaughton WK, Morris GP, Beck PL 1989 Inhibition of leukotriene synthesis markedly accelerates healing in a rat model of inflammatory bowel disease. Gastroenterology 96:29-36

24. Osman N, Adawi D, Ahrne S, Jeppsson B, Molin G 2004 Modulation of the effect of dextran sulfate sodium-induced acute colitis by the administration of different probiotic strains of Lactobacillus and Bifidobacterium. Dig Dis Sci 49:320-327

25. Araki Y, Andoh A, Takizawa J, Takizawa W, Fujiyama Y 2004 Clostridium butyricum, a probiotic derivative, suppresses dextran sulfate sodium-induced experimental colitis in rats. Int J Mol Med 13:577-580

26. Pavan S, Desreumaux P, Mercenier A 2003 Use of mouse models to evaluate the persistence, safety, and immune modulation capacities of lactic acid bacteria. Clin Diagn Lab Immunol 10:696-701

27. Lamine F, Fioramonti J, Bueno L, Nepveu F, Cauquil E, Lobysheva I, Eutamene H, Theodorou V 2004 Nitric oxide released by Lactobacillus farciminis improves TNBS-induced colitis in rats. Scand J Gastroenterol 39:37-45

28. Rolfe RD 2000 The role of probiotic cultures in the control of gastrointestinal health J Nutr 130:396S-402S

29. Rani B, Khetarpaul N 1998 Probiotic fermented food mixtures: possible applications in clinical anti-diarrhoea usage. Nutr Health 12:97-105

30. Tkalcic S, Zhao T, Harmon BG, Doyle MP, Brown CA, Zhao P 2003 Fecal shedding of enterohemorrhagic Escherichia coli in weaned calves following treatment with probiotic Escherichia coli. J Food Prot 66:1184-1189
31. Shu Q, Qu F, Gill HS 2001 Probiotic treatment using Bifidobacterium lactis HN019 reduces weanling diarrhea associated with rotavirus and Escherichia coli infection in a piglet model. J Pediatr Gastroenterol Nutr 33:171-177

32. Johnson E, Barthold SW 1979 The ultrastructure of transmissible murine colonic hyperplasia. Am J Pathol 97:291-313

33. Higgins LM, Frankel G, Douce G, Dougan G, MacDonald TT 1999 Citrobacter rodentium infection in mice elicits a mucosal Th1 cytokine response and lesions similar to those in murine inflammatory bowel disease. Infect Immun 67:3031-3039

34. Perdigon G, Alvarez S, Pesce de Ruiz Holgado A 1991 Immunoadjuvant activity of oral Lactobacillus casei: influence of dose on the secretory immune response and protective capacity in intestinal infections. J Dairy Res 58:485-496

35. Kaila M, Isolauri E, Soppi E, Virtanen E, Laine S, Arvilommi H 1992 Enhancement of the circulating antibody secreting cell response in human diarrhea by a human Lactobacillus strain. Pediatr Res 32:141-144

36. Shu Q, Gill HS 2002 Immune protection mediated by the probiotic Lactobacillus rhamnosus HN001 (DR20) against Escherichia coli O157:H7 infection in mice. FEMS Immunol Med Microbiol 34:59-64

37. Tejada-Simon MV, Lee JH, Ustunol Z, Pestka JJ 1999 Ingestion of yogurt containing Lactobacillus acidophilus and Bifidobacterium to potentiate immunoglobulin A responses to cholera toxin in mice. J Dairy Sci 82:649-660

38. Fukushima Y, Kawata Y, Mizumachi K, Kurisaki J, Mitsuoka T 1999 Effect of bifidobacteria feeding on fecal flora and production of immunoglobulins in lactating mouse. Int J Food Microbiol 46:193-197

39. Maaser C, Housley MP, Iimura M, Smith JR, Vallance BA, Finlay BB, Schreiber JR Varki NM, Kagnoff MF, Eckmann L 2004 Clearance of Citrobacter rodentium requires B cells but not secretory immunoglobulin A ( $\operatorname{Ig} A)$ or IgM antibodies. Infect Immun 72:3315-3324

40. Defrance T, Vanbervliet B, Briere F, Durand I, Rousset F, Banchereau J 1992 Interleukin 10 and transforming growth factor beta cooperate to induce anti-CD40activated naive human B cells to secrete immunoglobulin A. J Exp Med 175:671-782

41. von der Weid T, Bulliard C, Schiffrin EJ 2001 Induction by a lactic acid bacterium of a population of $\mathrm{CD} 4(+) \mathrm{T}$ cells with low proliferative capacity that produce transforming growth factor beta and interleukin-10. Clin Diagn Lab Immunol 8:695701

42. Pathmakanthan S, Li CK, Cowie J, Hawkey CJ 2004 Lactobacillus plantarum 299 beneficial in vitro immunomodulation in cells extracted from inflamed human colon. J Gastroenterol Hepatol 19:166-173

43. Malstrom C, James S 1998 Inhibition of murine splenic and mucosal lymphocyte function by enteric bacterial products. Infect Immun 66:3120-3127

44. Kim SH, Yang SJ, Koo HC, Bae WK, Kim JY, Park JH, Baek YJ, Park YH 2001 Inhibitory activity of Bifidobacterium longum HY8001 against Vero cytotoxin of Escherichia coli O157:H7. J Food Prot 64:1667-1673

45. Pena JA, Versalovic J 2003 Lactobacillus rhamnosus GG decreases TNF- $\alpha$ production in lipopolysaccharide-activated murine macrophages by a contact-independent mechanism. Cell Microbiol 5:277-285

46. Karlsson H, Larsson P, Wold AE, Rudin A 2004 Pattern of cytokine responses to gram-positive and gram-negative commensal bacteria is profoundly changed when monocytes differentiate into dendritic cells. Infect Immun 72:2671-2678

47. Simmons CP, Goncalves NS, Ghaem-Maghami M, Bajaj-Elliott M, Clare S, Neves B, Frankel G, Dougan G, MacDonald TT 2002 Impaired resistance and enhanced pathology during infection with a noninvasive, attaching-effacing enteric bacterial pathogen, Citrobacter rodentium, in mice lacking IL-12 or IFN-gamma. J Immunol 168:1804-1812 